\title{
Carnets
}

Revue électronique d'études françaises de l'APEF

Première Série - 3| 2011

$\mathrm{L}^{\prime}($ In)vraisemblable

\section{Aux confins de l'invraisemblable: voyage et fantaisie dans le récit court balzacien}

\section{Pedro Méndez}

\section{(2) OpenEdition}

Journals

\section{Édition électronique}

URL : http://journals.openedition.org/carnets/5996

DOI : 10.4000/carnets.5996

ISSN : 1646-7698

Éditeur

APEF

\section{Édition imprimée}

Date de publication : 2 janvier 2011

Pagination : 99-114

\section{Référence électronique}

Pedro Méndez, "Aux confins de l'invraisemblable: voyage et fantaisie dans le récit court balzacien », Carnets [En ligne], Première Série - 3 | 2011, mis en ligne le 18 juin 2018, consulté le 20 avril 2019.

URL : http://journals.openedition.org/carnets/5996; DOI : 10.4000/carnets.5996

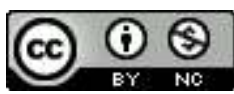

Carnets est mis à disposition selon les termes de la licence Creative Commons - Atribution - Pas d'utilisation commerciale 4.0 International. 


\title{
AUX CONFINS DE L'INVRAISEMBLABLE: \\ Voyage et fantaisie dans le récit court balzacien
}

PEDRO MÉNDEZ

Université de Murcia

psmendez@um.es

\begin{abstract}
Résumé
Face au Balzac réaliste, auteur engagé dans l'évocation vraisemblable d'une époque, et au Balzac philosophique et penseur, auteur de récits fantastiques, cet article se situe de l'autre côté, dans un domaine peu exploré de l'univers balzacien, celui de l'invraisemblable. Un insolite Balzac "voyageur" nous conduit dans un monde inédit de fantaisie et d'imagination où tout est possible. C'est en dehors du corpus de La Comédie Humaine, dans des œuvres marginales, que nous trouvons ces récits de voyages balzaciens (quatre seulement). Dans ce travail nous nous interrogeons sur les raisons de leur marginalité dans l'ensemble de la production balzacienne, tout en analysant leur signification et le rôle joué par la fantaisie et l'invraisemblable dans leur action narrative.
\end{abstract}

\begin{abstract}
In contrast to the general views of Balzac as a realistic author, philosopher and thinker, committed not only to the realistic depiction of his time but also to the creation of fantastic tales, this article focuses on an aspect of Balzac's universe which has been very little studied: the implausible. An unusual Balzac "traveler" takes us to an unknown world of fantasy and imagination where everything is possible. It is outside the corpus of La Comédie Humaine, in less important works, where we can find these journey short stories, which are only four. In this project we will wonder what the reasons for this marginal importance are in the context of the whole of his production. We will also analyze its meaning and the role that fantasy and the implausible play in his narrative.
\end{abstract}

Mots-clés: XIX ${ }^{\mathrm{e}}$ siècle, récit court balzacien, voyage, fantaisie, invraisemblable

Keywords: $19^{\text {th }}$ century, Balzac's short story, journey, fantasy, implausible 
Les motifs du voyage peuvent être variés. Dans sa réalisation peut intervenir un but scientifique, culturel, ludique ou un désir d'évasion personnelle. En tout cas, le voyage implique toujours un "aller" vers l'Autre, vers ce qui est différent, vers ce qui est étranger pour nous. Le récit de voyages raconte, donc, à partir d'approches très diverses, ce rapprochement de l'homme à une nouvelle réalité qu'il souhaite ou qu'il a besoin de connaître. Présent dans la création littéraire depuis ses origines, le voyage devient un sujet spécialement récurrent et significatif dans la seconde moitié du XVIII siècle et tout au long du siècle suivant, dû à des facteurs conjoncturels, comme l'expansion coloniale et l'amélioration qui commence à se produire dans les transports, facilitant les déplacements et réveillant l'intérêt de connaître d'autres gens, d'autres manières de vivre et de penser. Le romantisme français est attiré par l'exotisme d'autres époques comme celui de l'époque médiévale; d'autres lieux comme l'Italie, l'Allemagne, l'Espagne, les pays nordiques, l'Orient aussi. Le goût pour ce qui nous est inconnu, pour ce qui est perçu comme éloigné dans l'espace ou dans le temps, continue dans la période réaliste, de sorte que les voyageurs et les voyages abondent au XIX ${ }^{e}$ siècle non seulement sur le plan de la fiction littéraire, mais aussi dans celui de l'Histoire ou de la Géographie.

Pourtant, Balzac constitue une exception à un siècle où le voyage, non seulement comme fait fictionnel, mais aussi comme expérience personnelle, est à la mode parmi les écrivains. Bien qu'il ait visité des pays comme la Suisse, l'Ukraine, l'Allemagne, I'Italie ou la Hollande, ces voyages ne l'ont pas incité à pratiquer la littérature de voyages, de sorte que les motifs viatiques présents dans La Comédie Humaine n'ont finalement pas constitué des récits de voyages, si, dans le sens strict du terme, nous considérons le voyage comme le déplacement réel ou imaginaire d'un personnage, déplacement qui se transforme en anecdote déclencheuse et conductrice du récit. Balzac place la fiction de certains de ses textes hors de l'Hexagone, dans des pays comme l'Italie, l'Allemagne, la Norvège, la Belgique ou l'Espagne, mais l'évocation des expériences et des sensations que le déplacement crée chez le personnage manque d'entité comme axe narratif.

Le voyage est pour Balzac un objet intéressant, et peut-être même un des paramètres fondamentaux de la nouvelle société, prise dans le mouvement incessant de la civilisation urbaine, mais il ne constitue presque jamais une situation d'énonciation qui se prêterait au récit de ce qu'on a vu, de la manière dont on l'a vu, des impressions et pensées que l'on a eues. (Mozet \& Petitier, 2004: 6)

Le voyage est présent comme élément fictionnel qui perfectionne le portrait social de La Comédie Humaine, mais Balzac refuse de se plier aux conventions littéraires du genre et de transformer l'expérience du voyageur en vecteur narratif de l'œuvre. Par conséquent, "si 
la problématique contemporaine du récit de voyage apparaît dans La Comédie Humaine, c'est sous la forme d'une mise à distance" (Déruelle, 2003: 325-326), critiquant fréquemment un genre soumis en excès aux clichés ${ }^{1}$. Cette réticence à se plier aux dictées de ce qu'il considère une mode littéraire, "un exercice facile souvent réservé aux esprits mineurs et au menteurs" (Thérenty, 2004: 286), explique la dévalorisation narrative du voyage au sein de la création balzacienne. Seuls "les récits de voyages accompagnés de confidences et donc liés à l'exploration de l'intime" (Thérenty, 2004: 285) échappent à la parodie. Mais ce type de voyage "autobiographique et intime" (Thérenty, 2004: 288), même étant le seul légitime pour Balzac, est aussi refusé par l'auteur:

Il existe, en effet, dans le voyage une dimension confessionnelle semble-t-il insupportable à Balzac au point que son seul récit de voyage achevé [Voyage de Paris à Java] transforme en convention la nécessité autobiographique du genre, puisqu'il s'agit d'un récit de voyage imaginaire, sans voyage. [...]

Écrire un récit de voyage non parodique serait sans doute pour Balzac raconter l'expérience intraduisible d'un voyage à travers soi-même, hors de toutes les limites de la bienséance, hors de tous les codes génériques, exposition impudique, insupportable et d'ailleurs irréalisable [...]. (Thérenty, 2004: 290-291)

Cette distance avec laquelle Balzac envisage la thématique et la technique inhérentes au récit de voyages explique peut-être pourquoi les rares textes balzaciens que nous pouvons affirmer respectent pleinement les paramètres esthétiques du genre, sont restés hors du corpus de La Comédie Humaine. En outre, le récit de voyages s'articulant normalement dans des histoires d'une certaine longueur, Balzac se détourne de la tendance générale en donnant à ces œuvres la forme de textes courts ${ }^{2}$. Mais nous devons souligner un aspect qui nous semble être déterminant aussi de cet écartement. À l'exception de la Lettre sur Kiew, les récits de voyages balzaciens partagent une même caractéristique: le développement du thème du voyage dans le cadre d'un registre fictionnel qui emmène le lecteur dans le monde de l'imaginaire, de la fantaisie, de l'invraisemblable. Ce lien que l'auteur établit entre voyage et fantaisie ne termine pas de s'accommoder au système

\footnotetext{
${ }^{1}$ Selon Déruelle, le rejet du genre viatique est très évident quand il s'agit du voyage à des lieux situés hors de la France. Au contraire, Balzac reflète une certaine esthétique du voyage, quand il centre son regard sur la province française, pour la transformer en lieu romanesque et objet de description, en la faisant porteuse de l'exotisme cherché traditionnellement dans d'autres pays: "La province se voit attribuer les traits caractéristiques des pays étrangers. Balzac reprend parfois ce qui nous semble être un topos du voyage en France. Par exemple, point besoin d'aller en Amérique pour trouver le dépaysement: les Chouans, les paysans sont des Peaux-Rouges et des Mohicans. Le voyage dans les provinces françaises permet en quelque sorte au narrateur balzacien de faire l'économie des récits de voyages à l'étranger" (2003: 340).

2 Toutefois le récit bref n'est pas un genre considéré par lui comme marginal. Dans un siècle où la "nouvelle" connaît un essor sans précédent, Balzac est auteur de nombreux récits courts, la plupart publiés pour la première fois dans la presse, qui éprouve aussi un développement vertigineux au $\mathrm{XIX}^{\mathrm{e}}$ siècle et qui a contribué de manière décisive au triomphe des formes brèves.
} 
compositionnel qui dirige l'ensemble de son œuvre, présidé par le compromis de refléter de façon fidèle la réalité sociale et idéologique de son époque. Donc, si les récits ou les épisodes de type fantastique sont parfaitement imbriqués dans l'ensemble de La Comédie Humaine parce que le fonctionnement du fantastique a besoin d'un contexte réaliste, les narrations fantaisistes se situent, par contre, de l'autre côté, sur le plan du merveilleux, et elles s'éloignent de la représentation réaliste de l'œuvre balzacienne.

Outre la Lettre sur Kiev, quels sont les récits de Balzac proprement viatiques? Thérenty fait mention du Voyage de Paris à Java; mais nous pensons qu'il faut ajouter encore deux autres: Voyage d'un lion d'Afrique à Paris, et ce qui s'ensuivit et Voyage d'un moineau de Paris à la recherche du meilleur gouvernement. Voici, donc, les quatre récits balzaciens qui méritent sans aucun doute l'appellatif "viatique".

Étant moins intéressante pour l'objectif de notre étude, nous nous occuperons sommairement de la Lettre sur Kiev, œuvre de 1847. Thérenty (2004: 277) attribue à ce texte le privilège d'être, à côté de Voyage de Paris à Java, le seul exposant du genre viatique dans la production balzacienne. D'autres critiques, par contre, nient cette dimension parce que c'est un texte inachevé et trop court (Déruelle, 2003: 325). II s'agit du récit - dans une lettre dirigée à Armand Bertin, du Journal des Débats - du voyage qu'il a fait en Ukraine, dans les dernières années de sa vie, quand il était déjà malade, pour se marier avec celle qui avait été jusqu'à ce moment-là sa maîtresse, la comtesse polonaise Mme Hanska. S'agissant d'un texte autobiographique, c'est Balzac lui-même qui y intervient comme figure énonciative et ce qui y prédomine ce sont les impressions de voyage. Les rares éditeurs de ce texte l'ont considéré inachevé parce que, contrairement à ce que le titre nous ferait penser, il n'y a presque rien sur Kiev. Pourtant, selon Smethurst, si l'on analyse I'histoire en partant du schéma "moi-voyage-désir", elle peut être considérée comme parfaitement finie (2002: 270), de telle sorte que "la Lettre sur Kiev n'est pas un pèlerinage à la Rome orthodoxe [Kiev], mais un voyage vers l'être désiré, une quête du Graal, où le voyage même devienne une série d'épreuves pour l'être désirant” (2002: 275). Pour sa part, Thérenty explique la non conclusion du récit par la difficulté qu'éprouve Balzac de s'adonner à une narration intimiste et autobiographique: "La Lettre sur Kiew 's'inachève' sans doute parce qu'elle finirait par révéler le plus intime de l'écrivain, sa vie amoureuse" (2004: 290).

Quant à Voyage de Paris à Java, cette œuvre fut publiée dans la Revue de Paris, en novembre 1832. L'une des raisons qui sont signalées contre sa considération comme récit de voyages c'est qu'il s'agit d'un voyage imaginaire (Déruelle, 2003: 325) ${ }^{3}$. En effet, c'est un voyage imaginaire non seulement dans la fiction, mais aussi du point de vue des

\footnotetext{
${ }^{3}$ Marie Pinel (1996: 39-59) affirme, pour sa part, que Voyage de Paris à Java de Balzac, ainsi que Voyage autour de ma chambre (1794) et Expédition nocturne autour de ma chambre (1825), de Xavier de Maistre, parodient la littérature de voyages par le traitement de l'espace qui y existe.
} 
circonstances qui interviennent dans la composition du texte, puisqu'il s'agit d'une œuvre écrite seulement à partir du travail préalable de documentation de l'auteur ${ }^{4}$, qui ne s'est jamais rendu dans les pays asiatiques. Toutefois, il est évident que le récit de voyages se situe dans un terrain fluctuant où les frontières ne sont pas précisées:

Le récit de voyage souffre, en bref, de ce qu'on pourrait appeler un problème de territorialité. [...] On se rend bien vite compte de l'impossibilité d'isoler, dans un système taxinomique quelconque, une position stable qui serait le lieu de manifestation exclusif du récit de voyage. C'est que celui-ci ignore les frontières et déjoue les limites formelles que tout effort classificatoire cherche à instaurer. [...] Le récit de voyage, étant un peu partout, n'est nulle part en particulier. (Wetzel, 1992: 45)

Partant de cette prémisse, Voyage de Paris à Java, comme voyage imaginaire, n'est qu'une manière parmi d'autres de manifestation du genre. Voyage immobile, sa différence avec le voyage fictionnel qui est offert au lecteur comme expérience réellement vécue réside dans le fait que le déplacement dans l'espace n'est pas physique, mais il se produit dans la pensée du personnage: “[...] un rien m’embarquait fatalement, à travers le dédale des contemplations, sur un vaisseau fantastique, et faisait surgir les milles délices de mon voyage imaginaire" (Balzac, 1994: 8). Le récit, voisinant parfois les limites du fantastique, met en scène un personnage qui, obsédé par le désir de connaître l'Orient, se déplace de Paris à la région de la Touraine pour y essayer de se débarrasser de cette idée qui le tourmente. Mais il aura là-bas une vision qui l'incite finalement à entamer son voyage:

Je fus arrêté soudain, à la hauteur du vieux château de Valesne, par le fantôme du Gange, qui se dressa devant moi!... Les eaux de l'Indre s'étaient transformées en celles de ce vaste fleuve indien. Je pris un vieux saule pour un crocodile, et les masses de Saché pour les élégantes et sveltes constructions d'Asie... II y avait un commencement de folie à dénaturer ainsi les belles choses de mon pays: il fallait $y$ mettre ordre. Alors, tout fut dit. Je résolus de partir, malgré la rigueur de la saison, pour mon voyage dans les possessions de leurs Majestés Hollandaise et Britannique ${ }^{5}$. (Balzac, 1994: 9)

Animé par sa ferme décision, le personnage part vers Bordeaux où il pense embarquer vers l'Asie. Balzac profite pour réitérer à plusieurs reprises, comme nous avons signalé ci-dessus, sa position critique vers la littérature de voyages faite jusqu'à ce moment-

\footnotetext{
${ }^{4}$ Sur les sources qu'utilise Balzac pour la composition de Voyage de Paris à Java, voir Bui (2004: 261-264).

${ }^{5}$ Voir note 1.
} 
là. Le personnage narrateur exprime son désir de prendre distance à l'égard des topiques préétablis. "Il y eut dans ma détermination une sorte d'excentricity, dirait Lord Byron s'il vivait encore, qui ne me faisait ressembler à aucun des voyageurs vulgaires" (Balzac, 1994: 10). Le personnage part, donc, sans "remèdes contre le choléra-morbus, ni pacotille, ni tromblon, ni tente, ni lit de camp, rien enfin de ces mille choses inutiles aux voyageurs" (Balzac, 1994: 10). En outre, ce qu'il va raconter et la manière dont il va le raconter vont se détacher, volontaire et consciemment, de l'approche scientifique ou réaliste traditionnelle pour s'introduire pleinement dans le domaine de la fantaisie et de l'invraisemblable: "Je me promis surtout d'écrire mon voyage de manière à lui donner des teintes fabuleuses, afin d'être également lu par les savants, par les enfants, et cru par ceux qui croient tout ce qui est incroyable" (Balzac, 1994: 11).

Toutefois, le personnage n'arrivera jamais à Bordeaux. II fait un arrêt à Angoulême et c'est ici où finit le voyage réel et commence le voyage imaginaire à Java. Lors d'une soirée, accompagné de trois amis, après quelques points de suspension et sans qu'il y ait de transition, le récit du "non-voyage" par les terres orientales se déclenche.

Permettez-moi de supprimer toutes les niaiseries empreintes de personnalité par lesquelles mes devanciers commencent leurs relations. Pour abréger, lancez-vous sur-le-champ à travers l'Océan et les mers d'Asie, franchissez les espaces sur un brick assez bon voilier, et venons rapidement au fait: à Java, à mon île de prédilection... Si vous vous y plaisez, si mes observations vous intéressent, vous aurez économisé les ennuis de la route. (Balzac, 1994: 12)

En une trentaine de pages le lecteur assistera à une évocation confuse et désordonnée de la pensée débordée du personnage:

J'avoue que, pour un Européen, pour un poète surtout, aucune terre ne saurait être aussi délicieuse que l'île de Java!... Je vous parlerai des choses qui s'imprimèrent le plus vivement dans ma mémoire, mais sans ordre, au gré de mes souvenirs! Ce qu'un voyageur oublie est toujours peu de chose. $\mathrm{Si}$ je ne suis pas littérairement logique, je le serai relativement à l'ordre des impressions. (Balzac, 1994: 13)

"Je me suis laissé aller à mes fantaisies. J'ai vu tout en amateur et en poète" (Balzac, 1994: 20), dira-t-il après, en pleine extase des plaisirs javanais.

Tout d'abord, ce seront les femmes javanaises qui focaliseront l'attention du personnage. Dès le début il remarque le danger de tomber sous le charme de la beauté de ces créatures, femmes mortelles et vampiriques: 
Mais, à Java, la mort est dans l'air: elle plane autour de vous; elle est dans un sourire de femme, dans une œillade, dans un geste fascinateur, dans les ondulations d'une robe. Là, si vous avez la prétention d'aimer, de suivre vos penchants, vous périssez radicalement. (Balzac, 1994: 14)

Cependant, malgré le risque qu'entraine l'aventure amoureuse à Java, le personnage ne peut se détourner de la séduction fatale qu'exerce sur lui le charme exotique des femmes de Java:

Il est rare que les Européens résistent au spectacle de ces féeries. Quant à moi, j'y ai succombé malgré l'effroyable avertissement écrit sur le front de ces Javanaises. Presque toutes mariées cinq ou six fois, et cinq ou six fois veuves. Pour un artiste, qu'y a-t-il de plus tentant que de lutter avec ces femmes pâles, frêles, délicates, vampiriques?... (Balzac, 1994: 16)

Le mariage du personnage avec une javanaise attise en lui "les plaisirs du suicide", un "suicide par excès d'amour" (Balzac, 1994: 16). Mais il ne parvient pas à accomplir son projet parce que sa femme, qui était à l'origine de ses funestes pensées, meurt.

Je n'imaginais rien de plus poétique, de plus gracieux, que ces langueurs douces, ces prostrations complètes qui devaient m'amener insensiblement au néant. Eh bien! j'ai trouvé la réalisation de ces rêves insensés dans le mariage de Java. C'est l'amour dans toute sa poésie; l'amour ardent, l'amour ingrat, l'amour sans remords! [...]

Je fus sauvé de mon doux supplice par un accident. Ma Javanaise mourut, et je la regrettai bien vivement. (Balzac, 1994: 16-17)

Les digressions sur la faune et la flore de l'île aident à l'expression des sensations et des sentiments que la femme javanaise éveille au cœur du personnage:

Écouter les chants du bengali, respirer les volcamérias, en passant une main demimorte dans quelque chevelure javanaise, au frais, sous un ciel de feu, dans l'atmosphère humide que les Chinois savent produire en étendant de longues nattes en paille de riz, mouillées, devant les fenêtres de votre palais tranquille, tout tapissé de soie, de cachemires éclatants... Ah! cette vie est une débauche d'âme et de poésie, dont il n'existe d'image en aucune extase. Pour ceux qui l'ont goûtée, il n'y a plus ni arts, ni musique, ni chefs-d'œuvre! (Balzac, 1994: 22)

Dans un récit comme celui-ci, proche de la rêverie, la référence aux substances excitantes et leurs pouvoirs hallucinatoires ne saurait manquer. À Java le thé est l'une des 
plus consommées:

Le thé, pris à grandes doses et bu dans les contrées où, comme à Java, la feuille, fraîche encore, n'a rien perdu de ses précieux parfums, le thé vous verse tous les trésors de la mélancolie, les rêves, les projets du soir, même les conceptions inspirées par le café, même les jouissances de l'opium. [...] Votre état n'est pas le sommeil, mais une somnolence indécise semblable à la rêvasserie du matin. (Balzac, 1994: 27)

Mais la mort, évoquée à propos des femmes, revient dans le récit:

Toutes ces jouissances réunies, la Javanaise, les fleurs, les oiseaux, les parfums, le jour, l'air, cette poésie qui met une âme entière dans chaque sens, m'ont fait dire depuis mon retour des Indes:

- Heureux ceux qui vont mourir à Java!... (Balzac, 1994: 27)

Cette idée semble même devenir consubstantielle à bien des aspects qui définissent la culture javanaise. C'est le cas "de la merveille du pays, de l'upas, le seul arbre de cette espèce qui existe sur le globe, et dont les terribles produits jouent un si grand rôle dans les mœurs javanaises" (Balzac, 1994: 28). L'omniprésence de la mort ajoute parfois même un ton macabre à la rêverie narrative. Voici un fragment de la digression accordée à l'arbre vénéneux:

Figurez-vous une plaine d'ossements blanchis, ceinture digne de l'upas, témoignage de son pouvoir, malheureux atteints çà et là, quand ils se croyaient sauvés, la plupart amoncelés autour de l'arbre. Ces squelettes, frappés par le soleil des Indes, s'en renvoyaient capricieusement les rayons. Les jeux de la lumière, à travers ces dépouilles, produisaient des effets atroces. II y avait des têtes dont les yeux flamboyaient, des crânes qui semblaient maudire le ciel, et des dents qui mordaient encore!... (Balzac, 1994: 30)

L'arbre-fougère, les singes, les bisons, les crocodiles sont d'autres éléments javanais qui attirent l'attention du personnage. Leur présentation plonge le lecteur dans un jeu constant entre réalité et irréalité:

II n'y a ni paroles ni pinceaux pour dire ou pour peindre les mouvements, les physionomies, l'air fin ou spirituel, les lazzis de ces bonnes gens-là. Mais ce qui me fit tout à la fois rire et penser ce fut l'aspect des vieux singes blessés qui venaient en s'appuyant sur des cannes, et se traînant comme nos invalides errants sur le quai 
Bourbon. II ne leur manquait que des jambes de bois ou des bras en écharpe pour me donner une vue en raccourci de la nature humaine. (Balzac, 1994: 39)

"Un livre de voyage est une chimère dont l'imagination doit savoir enfourcher la croupe aérienne" (Balzac, 1994: 42). Nous sommes, donc, face à un voyage imaginaire, côtoyant la rêverie et l'incroyable, et riche en fantaisie; un voyage immobile, transformé en voyage à l'intérieur de nous-mêmes, dans le monde des émotions.

La comparaison de l'Orient avec l'Europe, de Java avec Paris, ayant été présente tout au long de la narration, celle-ci finit par une descente du "ciel", du "paradis" javanais à la réalité mondaine:

Ah! les Indes sont la patrie des voluptés!... Paris est, dit-on, la patrie de la pensée! Cette idée console. Cependant, la consolation serait plus complète si l'on pouvait rencontrer des Javanaises à Paris. Hélas! il n'y a que des demi-Javanaises, sans chevelures; puis les Parisiennes pensent, elles font de l'esprit, et la femme de l'Orient est une bête sublime. (Balzac, 1994: 44)

L'épisode javanais s'achève, comme il avait commencé, par des points de suspension. Le lecteur découvre alors que le narrateur du voyage à Java n'a pas été le personnage du récit cadre, mais l'un des amis dont il était accompagné à Angoulême. "- Merci, dis-je à ce voyageur; vous m'avez fait voir Java en m'épargnant le fret, les avaries, les tempêtes et la Javanaise" (Balzac, 1994: 45). La critique du genre viatique traditionnel est présente aussi à la fin de l'œuvre, où le personnage ironise sur le fait qu'un déplacement réel n'est pas nécessaire, pour connaître d'autres lieux; les livres ou le pouvoir de notre imagination peuvent suffire pour nous y transporter:

Pendant les premiers jours de mon arrivée à Paris, j'eus bien de la peine à me persuader que je n'avais point été à Java, tant ce voyageur avait vivement frappé mon imagination par ses récits. [...]

Enfin, s'il est possible d'avoir été plus réellement à Java que je n'y suis allé, je défie tous les voyageurs, anciens et modernes, de s'y être amusés plus que moi et de le connaître aussi bien, aussi mal que je le connais. Vrais ou faux, ces discours fantastiques m'ont inoculé toute la poésie indienne. (Balzac, 1994: 45)

Nous sommes face à un récit - devenu métaphore du pouvoir du mot pour nous faire voyager à d'autres espaces, à un autre temps - qui s'avère insolite dans l'ensemble de l'œuvre balzacienne par son irréalisme. À la fin, contrairement à ce qu'il pourrait supposer, le lecteur découvre que l'évocation de Java n'est qu'un voyage inexistant, non-fait, un rêve. 
Donc, par ce détachement du réel, le texte dépasse même la frontière du fantastique et se place définitivement dans une dimension fantaisiste.

Deux autres récits brefs, non considérés par la critique, mais qui sont aussi représentatifs du genre viatique, sont Voyage d'un lion d'Afrique à Paris, et ce qui s'ensuivit et Voyage d'un moineau de Paris à la recherche du meilleur gouvernement; tous deux maintenus, comme les textes précédents, en marge de La Comédie Humaine, probablement - nous l'avons déjà dit - parce que l'invraisemblance de leurs histoires ne convient pas à l'horizon social et idéologique de l'œuvre balzacienne. Ces deux œuvres - et trois autres encore $^{6}$ - ont été la contribution de Balzac à un volume collectif illustré ${ }^{7}$ que l'éditeur Hetzel a mis en marche en 1840 et dont le titre, dans un clin d'œil à l'auteur de La Comédie Humaine, était Scènes de la vie privée et publique des animaux. Les animaux peints par eux-mêmes et dessinés par un autre. Études de mœurs contemporaines ${ }^{8}$. La procédure suivie, comme cela est exposé dans la préface de l'œuvre, était celle-ci: Grandville élaborait les dessins et les auteurs qui collaboraient dans l'édition inventaient une histoire pour le personnage créé. L'œuvre est ainsi publiée en livraisons hebdomadaires, et atteint le chiffre de cinquante numéros. Outre Balzac et Hetzel lui-même ${ }^{9}$, d'autres auteurs comme Nodier et sa fille, Jules Janin, Louis Viardot et Musset y ont participé. La bonne amitié entre Hetzel et Balzac $^{10}$ a fait que celui-ci soit l'auteur qui a écrit le plus de récits pour l'anthologie - cinq au total, après l'éditeur qui en écrit dix ${ }^{11}$. Voyage d'un moineau de Paris à la recherche $d u$ meilleur gouvernement a été attribué à George Sand, mais en réalité l'œuvre a été composée par Balzac et Sand a seulement ajouté le dernier paragraphe du texte d'un ton plus révolutionnaire que celui que Balzac pouvait publiquement employer. Une lettre de remerciement dirigée par Sand à Hetzel, permet de conclure de manière évidente que I'histoire ne lui appartient pas. L'origine de cette confusion paraît se devoir à l'intérêt de Balzac de ne pas éveiller de soupçons de favoritisme envers lui par le fait d'avoir contribué à l'œuvre avec plus d'histoires que les autres collaborateurs.

Comme son titre laisse deviner, toutes les histoires de l'anthologie ont des animaux comme protagonistes, mais Hetzel affirme dans la préface de l'œuvre que celle-ci inaugure une conception nouvelle de la fable, puisque, au-delà de la critique sociale et politique qui a

\footnotetext{
${ }^{6}$ Peignes de cœur d'une chatte anglaise, Guide-Âne à l'usage des animaux qui veulent parvenir aux honneurs et Les amours de deux bêtes offertes en exemple aux gens d'esprit.

7 L'élaboration des dessins a été chargée à Grandville, qui avait illustré en 1838 les Fables de La Fontaine. II a repris ainsi sa facette comme caricaturiste politique, en critiquant la société de son temps.

${ }^{8}$ Dans notre rapprochement à la genèse de ces textes nous résumons les données que Rose Fortassier expose dans l'introduction et comptes rendus de Peines de cœur d'une chatte anglaise et autres scènes de la vie privée et publique des animaux. Cette édition (Flammarion, 1985) rassemble les cinq histoires balzaciennes mentionnées.

${ }^{9}$ Celui-ci adopte le pseudonyme de Stahl.

${ }^{10}$ Grâce à elle, Hetzel est devenu quelques années plus tard, avec Furne, le premier éditeur de La Comédie Humaine.

${ }^{11}$ Nodier et La Bédollière y ont apporté deux respectivement; Marie Menessier-Nodier, Alfred de Musset, Janin et Viardot, un; six autres textes ont été écrits par d'autres auteurs moins connus.
} 
traditionnellement caractérisé ce genre, il ne s'agit pas d'histoires racontées d'après l'optique humaine; les animaux sont à la fois les protagonistes, les narrateurs et les gestionnaires de l'action narrative:

Jusqu'à présent c'était l'homme qui s'occupait de l'animal; ici, c'est l'animal qui s'inquiète de l'homme, qui le juge en se jugeant lui-même. Le point de vue, comme on voit, est changé. Nous avons différé enfin en ceci que l'homme ne prend jamais la parole lui-même, qu'il la reçoit au contraire de l'animal devenu à son tour le juge, l'historien, le chroniqueur, et, si l'on veut, le chef d'emploi ${ }^{12}$.

Voyage d'un moineau de Paris à la recherche du meilleur gouvernement raconte à la première personne le périple d'un moineau qui vit dans la rue de Rivoli à Paris, dans la gouttière de la maison d'un illustre écrivain, défenseur des plus faibles. À cause de la lutte permanente entre riches et pauvres au sein de la société des moineaux parisiens, la communauté charge le protagoniste de voyager à travers le monde pour connaître les différentes formes de gouvernement qui existent et pouvoir ainsi décider laquelle est celle qui leur convient le plus:

On émit la proposition, approuvée à l'unanimité, d'envoyer un Moineau franc, impartial, observateur et instruit, à la recherche du Droit-Animal, et chargé de comparer les divers gouvernements. On me nomma. Malgré nos habitudes sédentaires, je partis en qualité de procureur général des Moineaux de Paris: que ne fait-on pas pour sa patrie! (Balzac, 1985: 96-97)

L'oiseau raconte les expériences vécues lors de son voyage: "[...] je mets la relation de mon voyage sur l'autel de la patrie, comme un renseignement diplomatique dû à la bonne foi d'un modeste philosophe ailé" (Balzac, 1985: 97).

Le moineau se rend premièrement à l'Île des Fourmis - l'Angleterre - mais ses enquêtes lui révèlent une organisation sociale injuste - "les Patriciennes naissent Patriciennes. Sans cela, où serait le miracle?" (Balzac, 1985: 99) - et une politique d'expansion qui ne finit pas de le convaincre:

Cette injuste agression était autorisée par le principe fondamental du gouvernement Formique dont la Charte a pour premier article: Ote-toi de là, que je m'y mette. Le second article porte en substance que ce qui convient à l'Empire Formique appartient à l'Empire Formique, et que quiconque s'oppose à ce que les sujets Formiques s'emparent devient l'ennemi du gouvernement Formique. (Balzac, 1985: 101)

\footnotetext{
${ }^{12}$ Cité par Fortassier (Balzac, 1985: 11).
} 
L'oiseau part "vraiment affligé de la perfection de cette oligarchie et de la hardiesse de son égoïsme" (Balzac, 1985: 103). La visite, ensuite, au royaume des abeilles lui permet de connaître le fonctionnement de la monarchie absolue de droit divin. Le moineau découvre aussi des inégalités dans ce système politique:

- Princesse, repris-je, il me semble que la mécanique à laquelle vous donnez le nom de peuple des Abeilles exclut toute liberté, vos Ouvrières font toujours absolument la même chose, et vous vivez, je le vois, d'après les coutumes égyptiennes.

- Cela est vrai, mais l'Ordre est une des plus belles choses. ORDRE PUBLIC, voilà notre devise, et nous la pratiquons [...]. La monarchie, c'est l'ordre, et l'ordre est absolu.

- L'ordre à votre profit, princesse. [...]

- Eh! que voulez-vous? l'État c'est moi. Sans moi, tout périrait. Partout où chacun discute l'ordre, il fait l'ordre à son image, et comme il y a autant d'ordres que d'opinions, il s'ensuit un constant désordre. (Balzac, 1985: 109)

Finalement, l'oiseau voyage à la république des loups, entre l'Ukraine et la Tartarie. Fondée sur les principes de liberté et d'égalité, cette organisation socialiste ne le satisfait pas non plus:

La fière république des Loups ne me satisfaisait plus entièrement. N'est pas, après tout, une triste condition, que de vivre uniquement de rapines? Si l'égalité entre Loups est une des plus sublimes conquêtes de l'esprit animal, la guerre du Loup à l'Homme, à l'Oiseau de proie, au Cheval et à l'Esclave, n'en reste pas moins en principe une abominable violation du droit des Bêtes.

[...] Je tombai donc dans d'horribles doutes sur la nature des gouvernements. Je vis que beaucoup apprendre, c'est amasser des doutes. (Balzac, 1985: 116)

À son retour à Paris, le petit oiseau découvre que le maître de la maison a été emprisonné ${ }^{13}$. L'histoire finit avec cette réponse de l'écrivain, qui est la seule contribution originale de George Sand au texte: “- D'où viens-tu, cher petit compagnon? s'écria-t-il. Si tu as vu beaucoup de pays, tu as dû voir beaucoup de souffrances qui ne cesseront que par la promulgation du code de la Fraternité" (Balzac, 1985: 117).

Voyage d'un lion d'Afrique à Paris et ce qui s'ensuivit raconte le voyage à Paris d'un lion de l'Atlas, prince héritier dans son royaume, et son laquais, un tigre. Le lion accomplit

\footnotetext{
${ }^{13}$ Fortassier (Balzac, 1985: 90) explique que, pour que l'histoire semble rédigée par George Sand, Balzac a créé ce personnage, derrière lequel se devine, en réalité, un ami de Sand, Lamennais, qui a été privé de liberté après la publication, en 1840, d'un pamphlet dans lequel il dénonçait les abus du pouvoir politique.
} 
ainsi la volonté de son père de voyager en Europe pour s'instruire et observer les hommes qui, leur ayant usurpé leur nom, se font appeler comme eux, "lions"14. "Si les Hommes ont la question d'Orient, les Lions ont la question d'Europe, où depuis quelque temps des Hommes usurpaient leur nom, leurs crinières et leurs habitudes de conquête" (Balzac, 1985: 126).

À son arrivée à Paris, le lion est emprisonné par le gouvernement français dans le jardin botanique. Confondu, il croit qu'on lui fait un grand accueil, mais un ours blanc - prince dans son royaume aussi - lui explique qu'en réalité il a été fait prisonnier. Le lion n'oublie pas l'objectif de sa mission et il questionne l'ours sur la raison pour laquelle les hommes utilisent leurs noms:

- Quel avantage les Hommes trouvent-ils, cher Prince Oursakoff, à prendre nos noms sans pouvoir prendre nos qualités?

- Il est plus facile d'avoir de l'esprit en se disant une Bête qu'en se donnant pour un Homme de talent! D'ailleurs, les Hommes ont toujours si bien senti notre supériorité, que, de tout temps, ils se sont servis de nous pour s'anoblir. Regardez les vieux blasons? Partout des Animaux! (Balzac, 1985: 128-129)

Le lion s'échappe et, accompagné de son tigre, il parcourt la ville à la recherche des lions de Paris. Tous les deux sont guidés par un chien. Dans la ville on célèbre le carnaval et les animaux marchent dans les rues sans attirer l'attention de personne. En chemin ils remarquent des aspects de la vie parisienne qui les surprennent négativement comme la pollution, la cuisine ou le système politique français:

À Paris, le roi règne et ne gouverne pas. Si vous ne comprenez pas ce système je vais vous l'expliquer. On rassemble par trois à quatre cents groupes tous les honnêtes gens du pays en leur disant de se représenter par un d'eux. On obtient quatre cent cinquante-neuf Hommes chargés de faire la loi. Ces hommes sont vraiment plaisants: ils croient que cette opération communique le talent, ils imaginent qu'en nommant un Homme d'un certain nom, il aura la capacité, la connaissance des affaires; qu'enfin le mot honnête Homme est synonyme de législateur, et qu'un Mouton devient un Lion en lui disant: Sois-le. (Balzac, 1985: 131-132)

Finalement le chien conduit ses compagnons dans un café où le lion africain connaît non seulement les lions et lionnes de Paris, mais aussi les loups-cerviers et les rats. Balzac joue dans la fiction avec l'acception argotique de ces termes: les capitalistes cherchant le bénéfice rapide sont connus comme loups-cerviers; les lions sont les hommes élégants et

\footnotetext{
${ }^{14}$ L'histoire est construite suivant la technique du récit encadré: il y a une histoire cadre qui ouvre et ferme l'action, où la narration est à la troisième personne; et une histoire centrale de type épistolaire, composée par les lettres que le lion et son laquais envoient au roi en Afrique, pour lui informer des étapes de son itinéraire.
} 
présomptueux; les lionnes sont leurs femmes; et les rats, leurs amants:

- [...] Puis, Monseigneur, le Lion de Paris se distingue moins par lui-même que par son Rat, et aucun Lion ne va sans son Rat. Pardon, Altesse, si je rapproche deux noms aussi peu faits pour se toucher, mais je parle la langue du pays.

- Quel est ce nouvel Animal?

- Un Rat, mon prince: c'est six aunes de mousseline qui dansent, et il n'y a rien de plus dangereux, parce que ces six aunes de mousseline parlent, mangent, se promènent, ont de caprices, et tant, qu'elles finissent par ronger la fortune des Lions, quelque chose comme trente mille écus de dettes qui ne se retrouvent plus! (Balzac, 1985: 135)

Le lion africain décide d'assister à un bal où finalement il a un entretien avec un lion parisien. II lui récrimine l'emploi inapproprié qu'on fait à Paris du nom "lion" et tous les deux entament alors une discussion après laquelle le lion africain, humilié par le lion parisien, décide de retourner dans son pays:

Son Altesse, Sire, jugea qu'elle n'avait plus rien à faire à Paris, que les Bêtes avaient grand tort de s'occuper des Hommes, qu'on pouvait les laisser sans crainte jouer avec leurs Rats, leurs Lionnes, leurs cannes, leurs joujoux dorés, leurs petites voitures et leurs gants; qu'il eût mieux valu qu'elle restât auprès de Votre Majesté, et qu'elle ferait bien de retourner au désert. (Balzac, 1985: 139)

Les deux œuvres partagent les mêmes caractéristiques. Nous avons pu constater qu'elles sont idéologiquement très riches, laissant deviner, derrière la fresque animale, une critique sociopolitique très marquée. Le type de personnages qu'elles mettent en scène des animaux qui parlent et qui sont capables de raisonner -, déplace la fiction narrative au terrain du merveilleux, de sorte que le lecteur est conscient dès le début qu'il pénètre dans une dimension différente de celle qui est régie par les lois naturelles, un monde où tout est possible. Pourtant, le voyage des personnages dans cet univers de fantaisie contient les éléments constitutifs qui interviennent généralement dans les récits de voyages traditionnels: il y a un itinéraire autour duquel est articulée l'histoire, les faits sont racontés suivant un ordre chronologique et spatial, et la narration à la première personne rend plus vraisemblable l'acte discursif du voyage.

Il se peut que nous soyons surpris par le fait que Balzac ait utilisé, dans ces histoires, des animaux comme personnages, parce que cela constitue un procédé unique et tout à fait exceptionnel chez lui. La critique et le portrait sociaux nous y étant offerts sous un masque animalier, toute possibilité de vraisemblance est détruite. En plus, comme Voyage de Paris à 
Java, ces histoires n'appartiennent pas à la typologie du fantastique développé par Balzac, lié à l'expression des théories scientifiques de son époque - aujourd'hui plutôt pseudoscientifiques - et à la révélation de ses convictions philosophiques plus personnelles. Une réflexion de l'auteur dans la préface de La Comédie Humaine peut nous aider à mettre en contexte ce type d'histoires dans son œuvre. Balzac y dit que "l'idée première de La Comédie Humaine fut d'abord chez moi comme un rêve, comme un de ces projets impossibles que l'on caresse et qu'on laisse s'envoler; [...] Cette idée vint d'une comparaison entre l'Humanité et l'Animalité" ${ }^{15}$ (Balzac, 1976-1981: I, 7). À la lumière de cette pensée on peut mieux comprendre la présence, dans La Comédie Humaine et hors d'elle, de nombreuses métaphores animales qui donnent naissance à un authentique bestiaire d'hommes-chien, d'hommes-cochon, etc. ${ }^{16}$. De la même manière, pourrions-nous arriver à expliquer aussi l'existence dans l'univers balzacien de ces histoires d'animaux humanisés, qui nous montrent nos vices et nos défauts.

Dans Voyage de Paris à Java l'itinéraire du personnage nous emporte du monde réel à un univers de sensations et de fantaisie. Dans Voyage d'un moineau de Paris à la recherche du meilleur gouvernement et dans Voyage d'un lion d'Afrique à Paris et ce qui s'ensuivit, le chemin est l'inverse; d'un monde où tout est possible, les animaux protagonistes nous rapprochent de notre propre réalité. Côtoyant constamment l'invraisemblable, le voyage chez Balzac - à la différence du voyage traditionnel - essaye de nous conduire vers un inconnu qui ne se trouve pas nécessairement éloigné de nous dans l'espace ou dans le temps, mais il se trouve plutôt dans la force interne de l'individu pour appréhender le monde d'un autre regard. Moyen d'éloignement de la réalité, de cette quotidienneté si bien décrite dans les différentes Scènes de La Comédie Humaine, le voyage balzacien déplace le lecteur vers une nouvelle dimension, le monde de l'imagination et de l'incroyable, où l'acte de se comprendre lui-même et de comprendre ce qui l'entoure semble devenir moins problématique pour Balzac.

\footnotetext{
${ }^{15}$ Selon Castex "le jeune Balzac a pressenti que [...] 'la société ressemblait à la Nature' et qu'il existait des espèces sociales, comme il y a des espèces zoologiques. Ainsi naquit dans son esprit l'idée d'un système de la Société, analogue au système de la Nature" (Balzac, 1976-1981: I, XI).

${ }^{16}$ Sur ce thème, voir Thérien (1979).
} 


\section{Bibliographie}

BALZAC, Honoré de (1976-1981). La Comédie Humaine. Paris: Gallimard, coll. "Bibliothèque de La Pléiade", 12 vols.

BALZAC, Honoré de (1994). Voyage de Paris à Java. La Rochelle: Rumeur des Ages.

BALZAC, Honoré de (1985). Peines de cœur d'une chatte anglaise et autres scènes de la vie privée et publique des animaux. Introduction, notices, bibliographie, chronologie par Rose Fortassier. Paris: Flammarion.

BuI, Véronique (2004). "D'Issoudun à Java ou de l'Inde en Indre-et-Loire". In: Nicole Mozet et Paule Petitier (éds.), Balzac voyageur. Parcours, déplacements, mutations. Tours: Université François Rabelais, pp. 259-275.

Deruelle, Aude (2003). "L'Égypte, c'est tout sables'. Balzac et le récit de voyage”. In: Alain Guyot et Chantal Massol (éds.), Voyager en France au temps du Romantisme: Poétique, esthétique, idéologie. Grenoble: ELLUG, pp. 325-341.

Mozet, Nicole et Petitier, Paule (2004). "Présentation". In: Nicole Mozet et Paule Petitier (éds.), Balzac voyageur. Parcours, déplacements, mutations. Tours: Université François Rabelais, pp. 310.

PINEL, Marie (1996). "Le voyage immobile ou le traitement de l'espace dans les avatars du récit de voyage. Xavier de Maistre: Voyage autour de ma chambre, 1794, et Expédition nocturne autour de ma chambre, 1825; Balzac: Voyage de Paris à Java, 1832". In: Les Cahiers du SEL, no 1, pp. 3959.

SMETHURST, Colin (2002). "De Java à Kiev: Le moi du voyageur". In: L'année balzacienne, pp. 269278.

THERENTY, Marie-Ève (2004). "Itinéraires d'écrivains et récits de voyage dans La Comédie Humaine. Pourquoi Balzac n'a-t-il pas écrit de récit de voyage?". In: Nicole Mozet et Paule Petitier (éds.), Balzac voyageur. Parcours, déplacements, mutations. Tours: Université François Rabelais, pp. 277-292.

THERIEN, Michel (1979). "Métaphores animales et écriture balzacienne: le portrait et la description". In: L'année balzacienne, pp. 193-208.

WETZEL, Andreas (1992). Partir sans partir. Le récit de voyage littéraire au XIXe siècle. Toronto: Éditions Paratexte. 